\title{
Light and Dark: Fluorescent and Electron Dense Labeling for Neuronal Cells Using a Novel Viral Vector
}

Connon I. Thomas ${ }^{1}$, Naomi Kamasawa ${ }^{1}$, Satoko Okayama $^{2}$, Christian Keine ${ }^{3}$, Rachel Satterfield ${ }^{2}$, Debbie Guerrero-Given ${ }^{1}$ and Samuel M. Young, $\mathrm{Jr}^{2,3,4}$

${ }^{1 .}$ Electron Microscopy Core Facility, Max Planck Florida Institute for Neuroscience, Jupiter, FL.

2. Molecular Mechanisms of Synaptic Function, Max Planck Florida Institute for Neuroscience, Jupiter, FL.

3. Department of Anatomy and Cell Biology Iowa Neuroscience Institute, and Aging Mind Brain Initiative, University of Iowa, Iowa City, Iowa.

4. Department of Otolaryngology, Iowa Neuroscience Institute, and Aging Mind Brain Initiative, University of Iowa, Iowa City, Iowa.

Many tools for simultaneous labeling of neurons for light (LM) and electron microscopy (EM) are available, but a method that provides strong fluorescence, electron dense signal, and is also convenient to use is hard to find. Currently available tools are largely limited in scope by their incompatibility with certain fixation conditions, reliance on harsh detergents, complex procedures, or specific imaging modalities [1-6].

We created a Helper-dependent Adenoviral vector containing EGFP and a modified peroxidase targeted to mitochondria (MitoAPEX2), and transduced globular bushy cells from the anteroventral cochlear nucleus of neo-natal mice. Several days after transduction, cells exhibited strong fluorescence, and electron dense mitochondria were evident under EM. The axons of these cells terminate in a giant axosomatic glutamatergic synaptic terminal called the calyx of Held, a model for study by electrophysiology and LM/EM. Due to the robust expression of EGFP and peroxidase labeling, strong fixation of tissue with the addition of glutaraldehyde could be achieved, making this an excellent tool for analysis of subsynaptic ultrastructure.

We began by confirming exogenous expression of EGFP-MitoAPEX2 had no effect on synaptic ultrastructure or function at the calyx of Held by TEM analysis and electrophysiological study. Active zone length and synaptic vesicle docking were not affected by the expression. In addition, EPSC amplitude, ready releasable pool size, replenishment rate, and release probability remained unchanged. We further confirmed co-expression of the markers at the terminal by post-embedding immuno-gold labeling for EGFP in epoxy resin in conjunction with DAB labeling of mitochondria. Immunogold labeling for EGFP was found only in the MitoAPEX2 positive terminals and gold particle density was significantly higher than background noise.

Furthermore, we applied this co-expression system to modern, high-throughput methods of EM such as serial section SEM (ssSEM) by automated tape-collecting ultramicrotome and serial block face SEM (SBF-SEM). For high resolution analysis of partial volumes, we find ssSEM is most appropriate due to the ability to perform this imaging method using high beam dwell times. SBF-SEM, with its fast imaging and straightforward image alignment, is ideal for reconstructing large volumes of tissue that contain multiple whole calyx of Held terminals. We suggest that it has potential correlative use in the study of how genetic perturbation affects function and structure of neurons [7]. 
References:

[1] J Lichtman and W Denk, Science 332 (2011), p. 618.

[2] X Shu et al, PLoS Biology 9(4) (2011), p. e1001041.

[3] M Ellisman et al, Methods in Cell Biology 111 (2012), p. 139.

[4] H Horstmann, M Vasileva, T Kuner, PLoS One 8 (2013), p. e64764.

[5] S Lam et al, Nature methods 12 (2015), p. 51.

[6] R Shigemoto, M Joesch, Wiley Interdisciplinary Reviews: Developmental Biology 6(6) (2017), p. e288.

[7] The authors thank the specialists of Applications and Services from RMC/Boeckeler, Fibics, and Carl Zeiss Microscopy for their intensive support.
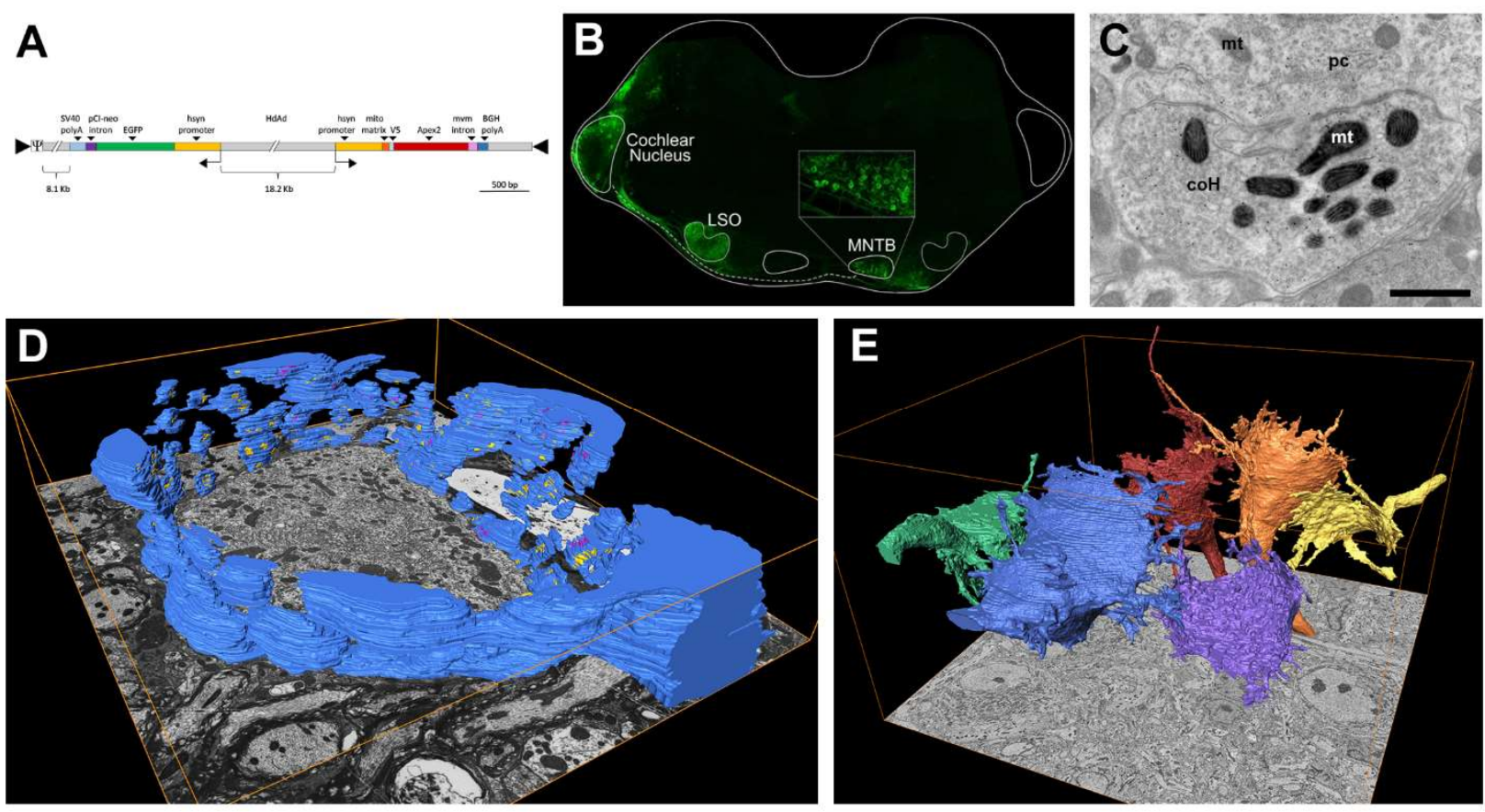

Figure 1. EGFP-MitoAPEX2 labeling scheme of the calyx of Held terminal. (A) DNA construct encoding EGFP and mitochondrial matrix-targeted APEX2. (B) Sectioned brainstem showing stereotactic injection of HdAd vector to the anteroventral cochlear nucleus. Following injection at P1, EGFP fluorescence can be seen in calyx terminals of the contralateral medial nucleus of the trapezoid body (MNTB). (C) Double labeling of the presynaptic calyx terminal. APEX2 targeted to the mitochondrial matrix provides local deposition of osmium tetroxide following application of DAB. This is followed by post-embedding immuno-gold labeling of GFP in epoxy sections with antibody conjugated to $12 \mathrm{~nm}$ immunogold particles. $\mathrm{mt}$, mitochondria; coH, calyx of Held; pc, principal cell. Scale bar: $1 \mu \mathrm{m}$ (D) Partial 3D reconstruction from 100 serial sections obtained with the ssSEM workflow showing active zones (yellow) and puncta adherentia (pink). (E) Six whole transduced calyx terminals reconstructed from a single volume of SBF-SEM, distinguished from surrounding negative terminals. 